\title{
Inhaltsverzeichnis nach Beitragsarten
}

\section{Abhandlungen}

Stephan Meder/Christoph-Eric Mecke: Legitimation güterrechtlicher Teilhabe im Spannungsverhältnis von Solidarität und Individualisierung

Thorsten Keiser: Vom familienrechtlichen Vertrag sui generis zum lebensgemeinschaftlichen Kooperationsvertrag - eine gelungene Rechtsfortbildung?

Friederike Wapler: Familie und Familienschutz im Wandel - zur Entwicklung des Familienbegriffs im öffentlichen Recht

Edward Schramm: Sozialer Wandel der Familie im Strafrecht

Christine Morgenstern: Ein Recht auf Hoffnung aus Art. 3 EMRK: Lebenslange Freiheitsstrafen in Europa

Volker Boehme-Neßler: Prekäre Balance: Überlegungen zum heiklen Verhältnis von Richtern und Gutachtern

Martin Klose: Vindikationsverjährung: Gewogen und für verfassungswidrig befunden!

Markus Ludwigs: Die Energiewende im Zeichen des Europa- und Verfassungsrechts

Andreas Engert: Private Normsetzungsmacht: Die Standardisierung von Regelungen im Markt als Form der Fremdbestimmung

Hans-Heinrich Trute: In der Grauzone: Akkreditierung zwischen öffentlichrechtlicher und privatrechtlicher Rechtsdurchsetzung

Jens Kersten: Das Anthropozän-Konzept

Lothar Michael: Die verfassungswandelnde Gewalt $\quad 426$

Carsten Bäcker: Nichtbegründetes Nichtannehmen $\quad 481$

Hanjo Hamann: Die Fußnote, das unbekannte Wesen - Potential und Grenzen $\quad 501$ juristischer Zitationsanalyse

\section{Rezensionsaufsätze}

Tilmann Altwicker: Die Zukunft des Völkerrechts - Rechtsökonomisch betrachtet (Trachtman)

Michael Fehling: Zum Zusammenspiel von Recht und Ökonomik bei Effizienzanforderungen an Unternehmen (Ludwigs)

Moritz Brinkmann: Normdurchsetzung durch Privatrecht (Pölzig)

Mario Bachmann: Verhältnismäßigkeit und Grundrechtsschutz im Präventionsstrafrecht (Kaspar) 
Jochen Bung: Das Unrecht des Bürgers (Pawlik)

\section{Tagungen und ihre Folgen}

Reinmar Wolff: Die Macht des gesellschaftsrechtlichen Gesetzgebers- zur

Ausgestaltung der Vereinigungsfreiheit (Art. 9 Abs. 1 GG) und ihren

Grenzen

Michaela Hailbronner/Michael Riegner: Das letzte Wort im Vergleich

Udo Moewes: L'État, c'est quoi? - Staatsgewalt im Wandel

Volker Wiese: „Methoden des Privatrechts“

Claudio Franzius: Parteienwissenschaften - interdisziplinär?

Marc Engelhart: Paradigmenwechsel im Strafrecht: Die Notwendigkeit einer „Neuen Strafrechtswissenschaft"?

Johannes W. Flume: 24. Tagung der Gesellschaft Junger

Zivilrechtswissenschaftler

Daniel Hlava: „Arbeitsrecht - für wen und wofür?“

\section{Rahmenbedingungen von Forschung und Lehre}

Matthias Klatt/Tina Winter: Der Hörsaal als Lernraum

Denis Basak/Marc Reiß/Roland Schimmel: Wissenschaftlichkeit der

Rechtswissenschaft?

\section{Autorenverzeichnis}

Altwicker, Tilmann: Die Zukunft des Völkerrechts - Rechtsökonomisch betrachtet (Trachtman)

Bachmann, Mario: Verhältnismäßigkeit und Grundrechtsschutz im Präventionsstrafrecht (Kaspar)

Bäcker, Karsten: Nichtbegründetes Nichtannehmen

Basak, Denis/Marc Reiß/Roland Schimmel: Wissenschaftlichkeit der

Rechtswissenschaft?

Boehme-Neßler, Volker: Prekäre Balance: Überlegungen zum heiklen

Verhältnis von Richtern und Gutachtern

Brinkmann, Moritz: Normdurchsetzung durch Privatrecht (Pölzig)

Bung, Jochen: Das Unrecht des Bürgers (Pawlik) 